\title{
Left Atrial Appendage Closure with Double Watchman Devices: A Case Report
}

\author{
Faisal Alsmadi, MBBS, FRCPC, FACP, FACC, FSCAI, MHRS, \\ Muhammad Azam Shah, MBBS, FCPS (Cardiology) ${ }^{*}$, Mohammad Bara Qattea, MD
}

Department of Cardiology, King Salman Heart Center, King Fahad Medical City, Riyadh, Saudi Arabia

\begin{abstract}
The risk of cardioembolic stroke is high in patients with atrial fibrillation. Antiplatelet agents, vitamin $\mathrm{K}$ antagonists, and new oral anticoagulants (NOACs) are effectively used to reduce the risk of thromboembolism in high-risk patients. However, increased risks of life-threatening bleeding and narrow therapeutic indexes result in inadequate utilization of these therapies. There is growing practice and evidences in favor of closing the left atrial appendage (LAA) percutaneously by using different devices in patients with either contraindicated or difficult anticoagulation. We report a rare case of a 75-year-old man with atrial fibrillation, high thromboembolic risk (CHADSVASc score of 4), and high bleeding risk score (HASBLED score of 4). He underwent LAA closure using 2 LAA percutaneous closure devices (Watchman) due to bilobed LAA. Considering the great variability in shape and size of the LAA, a single device may not always cover the whole ostium, which leads to residual leaks that can cause a nidus for thrombus formation. Although it technically sounds feasible, a few challenges are associated with double-device implantation. Sealing of the bilobed LAA is technically possible, especially with favorable anatomy, which includes totally separated bodies of both lobes with adequate body sizes.
\end{abstract}

Copyright $\odot 2019$ Science International Corp.

Key Words

Atrial fibrillation - Left atrial appendage closure • WATCHMAN device • Anticoagulation

Fax +1 2037853346

E-Mail: jshd@scienceinternational.org

http://structuralheartdisease.org/
(C) 2019 Journal of Structural Heart Disease Published by Science International Corp. ISSN 2326-4004

Accessible online at: http://structuralheartdisease.org/
Introduction

Prevention of cardioembolic stroke is one of the key goals in the treatment of patients with atrial fibrillation (AF). The risk of embolic stroke with nonvalvular AF is 5.6 times higher [1]. Anticoagulants such as vitamin $\mathrm{K}$ antagonists (VKA) and new oral anticoagulants (NOACs) are used effectively to reduce the risk of thromboembolism [2]. However, increased risks of mortality, bleeding, and narrow therapeutic indexes result in inadequate utilization of these therapies. There is growing practice and evidence in favor of closing the left atrial (LA) appendage (LAA) percutaneously by using different devices in patients with either contraindicated or difficult anticoagulation. Considering the great variability in the shape of the LAA, sometimes, a single device may not cover the whole ostium, which leads to residual leaks that can cause a nidus for thrombus formation. A previous report on double-device LAA closure using an Amplatzer cardiac plug (ACP) showed favorable results at follow-up [3]. We report a rare case of a 75-year-old man with $A F$ and high thromboembolic risk who underwent LAA closure using double Watchman devices.

\section{Case Summary}

A 75-year-old man who had diabetes, hypertension, hypothyroidism, a post-coronary artery bypass grafting 32 years before, chronic kidney disease (glo-

* Corresponding Author:

Muhammad Azam Shah, MBBS, FCPS (Cardiology)

Department of Cardiology

King Salman Heart Center

Dabab Street, Sulaimaniya, PO Box 59046, Riyadh 11525, Riyadh, Saudi Arabia

Tel. +00566539417315; E-Mail: azamshah165@hotmail.com 


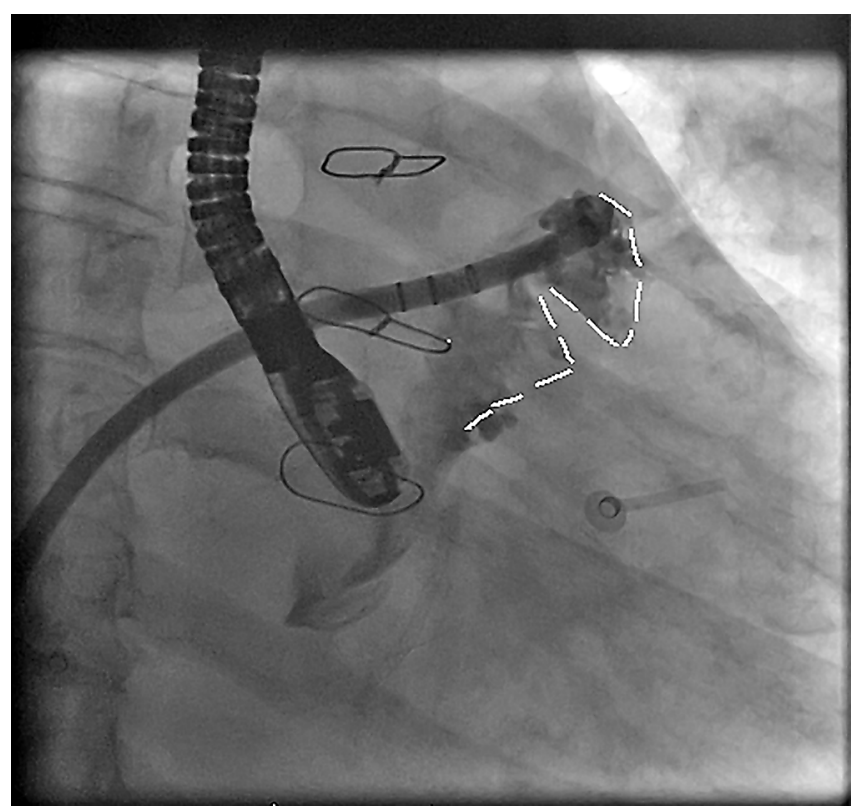

Figure 1. On contrast injection (RAO 200, CAU 180) showing bilobed LAA morphology with very wide ostium.

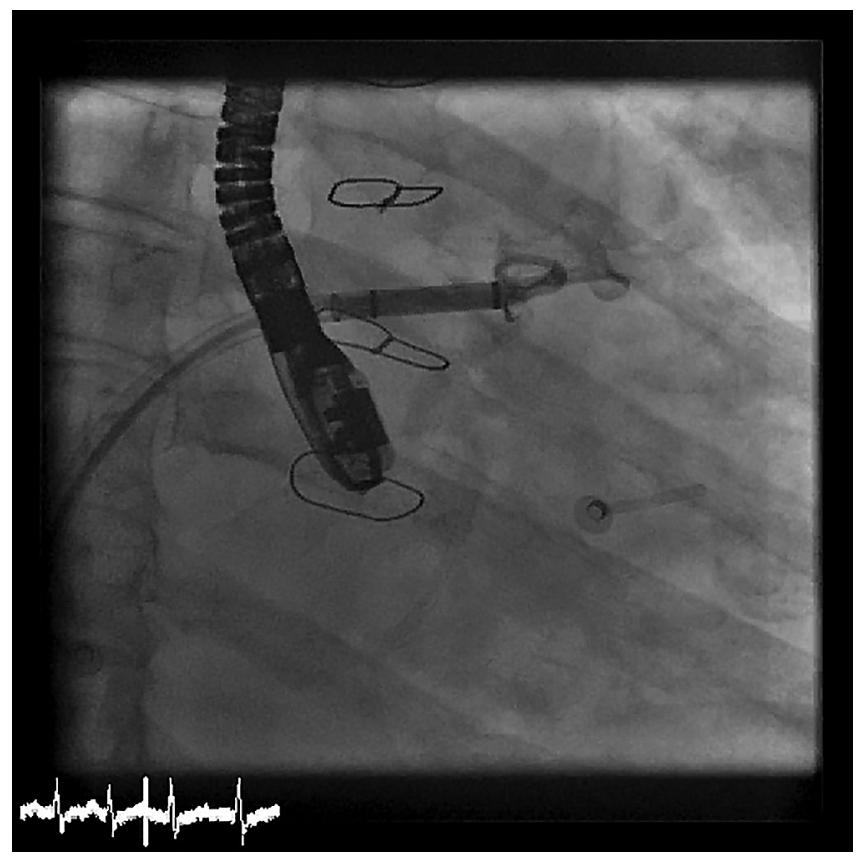

Video 1. On contrast injection (RAO 200, CAU 180) showing bilobed LAA morphology with very wide ostium. View supplemental video at https://doi.org/10.12945/j.jshd.2019.010.18.sup.01.

merular filtration rate $=48 \mathrm{~mL} / \mathrm{min}$ ) with an ejection fraction of approximately $40 \%$ underwent a permanent AF (CHADSVASc and HASBLED scores of 4). He underwent anticoagulation using rivaroxaban $15 \mathrm{mg}$.
He was undergoing follow-up as an outpatient because of chronic anemia and heart failure symptoms, and was admitted at our hospital with lower gastrointestinal (GI) bleeding and decompensated heart failure. The patient was hemodynamically stable and had bilateral crepitation at the bases of the lungs along with mild pedal edema on physical examination. His hemoglobin level was $8.4 \mathrm{~g} / \mathrm{dL}$ and serum creatinine was $193 \mu \mathrm{mol} / \mathrm{L}$. Colonoscopy revealed two colonic polyps. Considering difficult anticoagulation due to the persistent anemia and lower Gl bleeding, he was referred for LAA device closure.

Echocardiography documented a severely dilated LA (indexed LA volume, $63 \mathrm{~mL} / \mathrm{m}^{2}$ ). Pre-procedural transesophageal echocardiography revealed a LAA ostial diameter of $20 \mathrm{~mm}$ with a depth of $27 \mathrm{~mm}$. The procedure was performed under general anesthesia. A right femoral venous access was used after the transseptal LA puncture pressure was measured. A double curved Watchman access sheath was positioned in the LAA over a pigtail catheter. On contrast injection (RAO 20, CAU 18), a bilobed LAA morphology with very wide ostium was observed (Figure 1, Video 1). After multiple measurements, implantation of two devices was planned as a single device was thought to be inadequate to cover the whole ostium. By using a 14-F Watchman delivery system, a 33-mm Watchman device (Atritech Inc., Boston Scientific, Plymouth, MA) was selected and implanted successfully in the anterior lobe. The device was released after confirming the stability by using a tug test. Both angiography and echocardiography revealed a significantly sized lobe posteriorly (Figures 2 and 3, Videos 2 and 3), which was sealed using a 21-mm Watchman device (Kissing Watchman) by using the same delivery system (Videos 4 and 5). Residual peri-device leaks were excluded, and the stability of both devices was assessed (Figures 4 and 5). The patient was extubated and transferred to the critical care unit for recovery. He was discharged afterward with clopidogrel $75 \mathrm{mg}$ daily and an adjusted dose of warfarin. He underwent follow-up transesophageal echocardiography (TEE) after 6 weeks of the procedure, which showed well-seated watchman devices with trivial peri-device leakage (Video 6). No other complications were observed. The patient was advised to discontinue anticoagulation. 


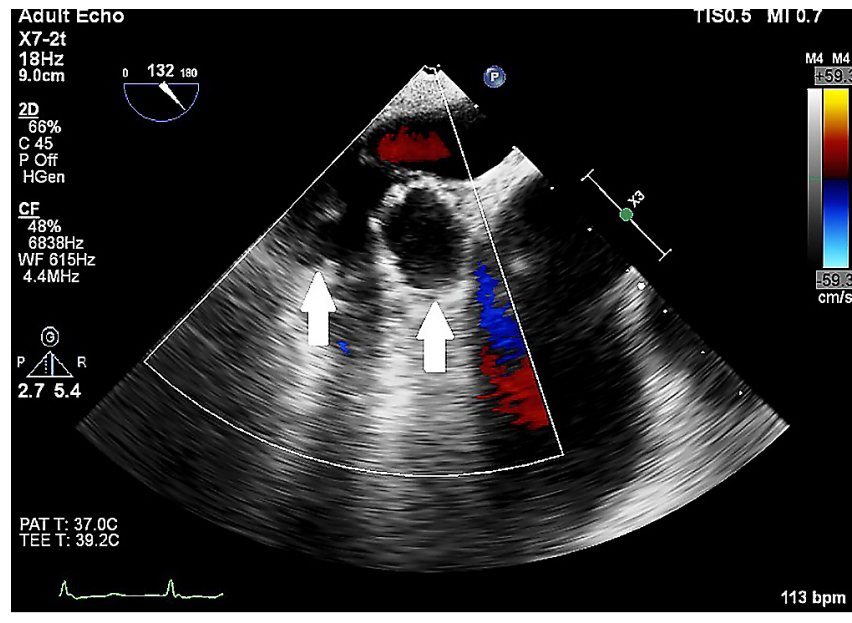

Figure 2. TEE image at 132 degrees with color showing implanted WATCHMAN device in anterior lobe with significant gap and flow posteriorly.

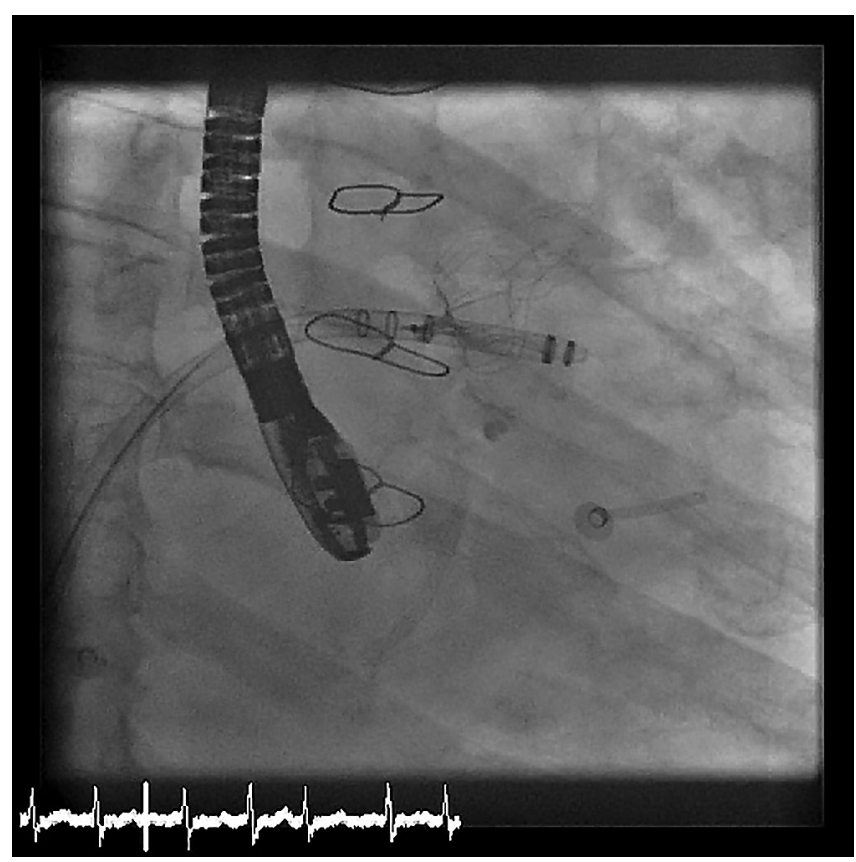

Video 2. Contrast injection after implantation of WATCHMAN device anteriorly showed posterior lobe with good size ostium. View supplemental video at https://doi.org/10.12945/j. jshd.2019.010.18.sup.02.

\section{Discussion}

AF is one of the most common cardiac arrhythmias (1-2\% in Western countries) [4]. More than $15 \%$ of cerebral ischemia cases are related to AF [5]. Almost $90 \%$ of atrial thrombi are formed in the LAA in patients with nonvalvular AF [6]. Systemic antico-

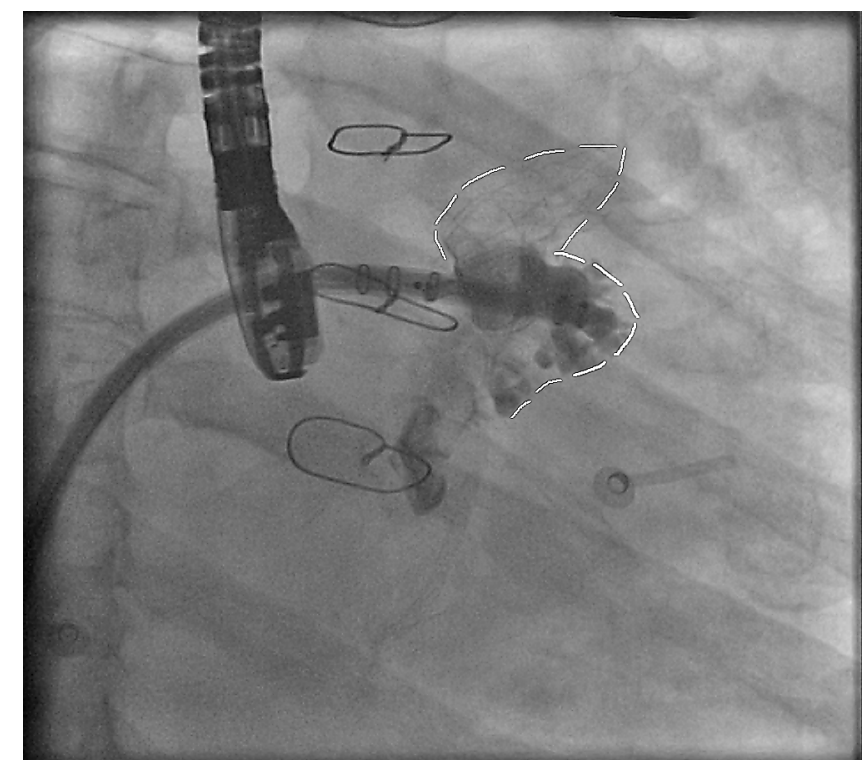

Figure 3. Contrast injection after implantation of WATCHMAN device anteriorly showed posterior lobe with good size ostium.

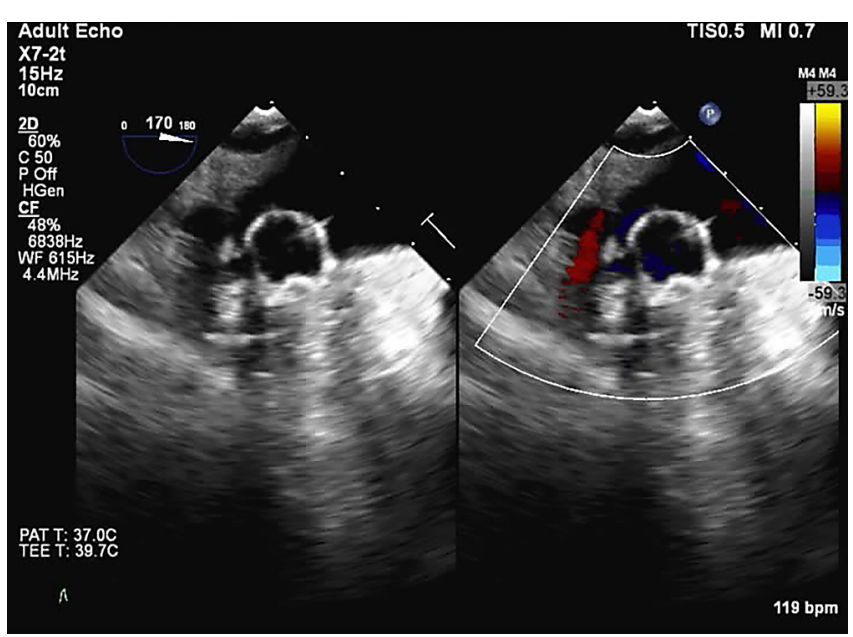

Video 3. TEE image at 170 degrees with color showing implanted WATCHMAN device in anterior lobe with significant gap and flow posteriorly. View supplemental video at https://doi. org/10.12945/j.jshd.2019.010.18.sup.03.

agulation is the therapy of choice to reduce the risk of thromboembolism in AF, but studies have shown that only few patients receive such therapies despite being indicated for multiple reasons, including complications and noncompliance [7]. Considering these limitations, percutaneous closure of the LAA is becoming increasingly popular in selected patients. 


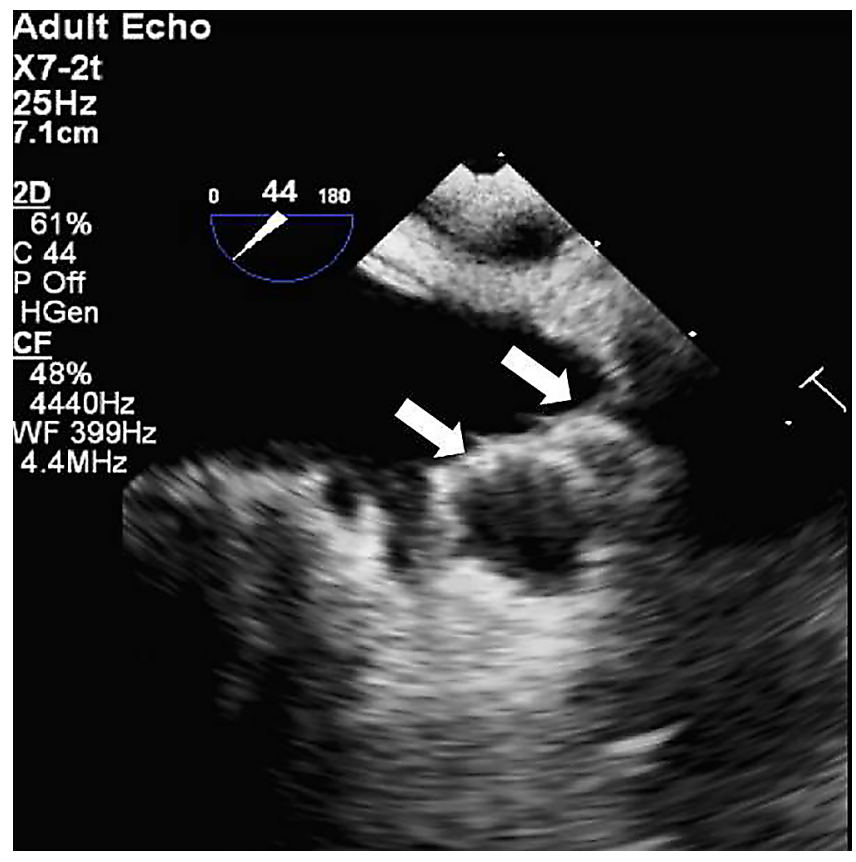

Figure 4. TEE $2 D$ image at 44 degrees showing two WATCHMAN devices implanted side by side.

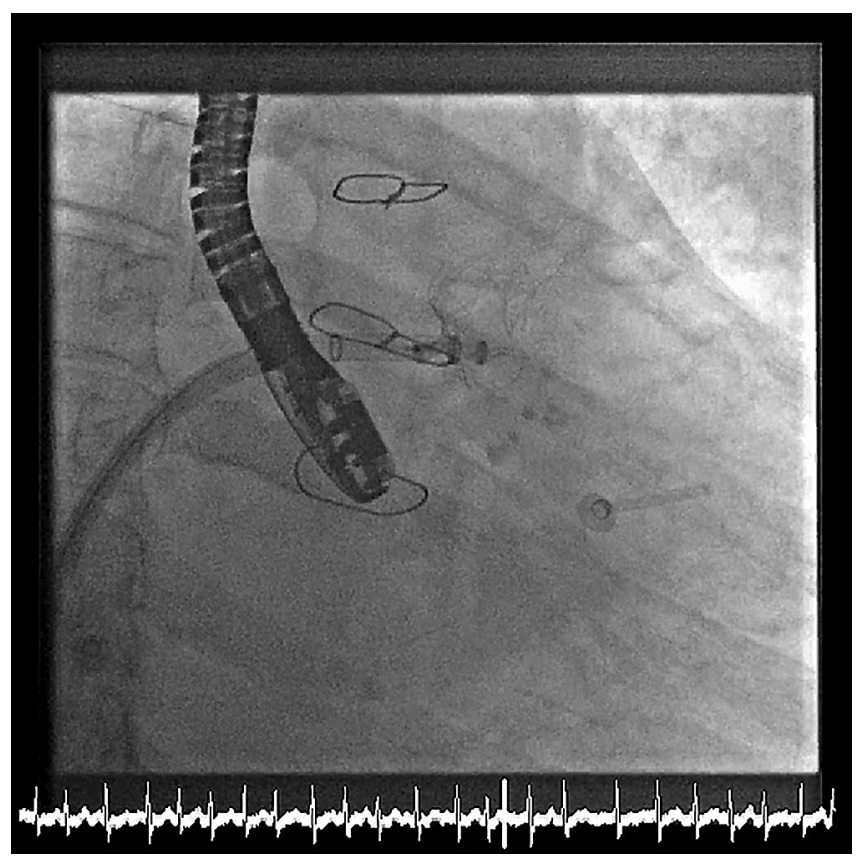

Video 4. Cine clip showing release of second WATCHMAN device. View supplemental video at https://doi.org/10.12945/j. jshd.2019.010.18.sup.04.

Since the introduction of LAA closure devices about 15 years before, multiple types and shapes of devices have been introduced and tested, but the ACP and Watchman system are the most widely used. Multiple

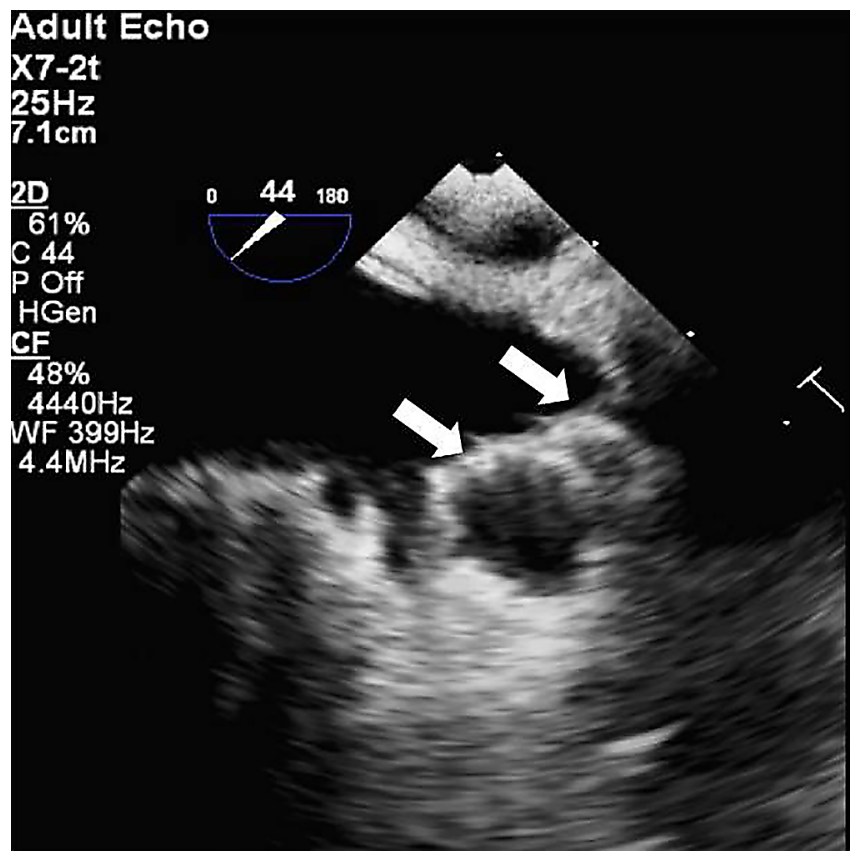

Figure 5. Cine image showing two WATCHMAN devices (Kissing WATCHMAN)

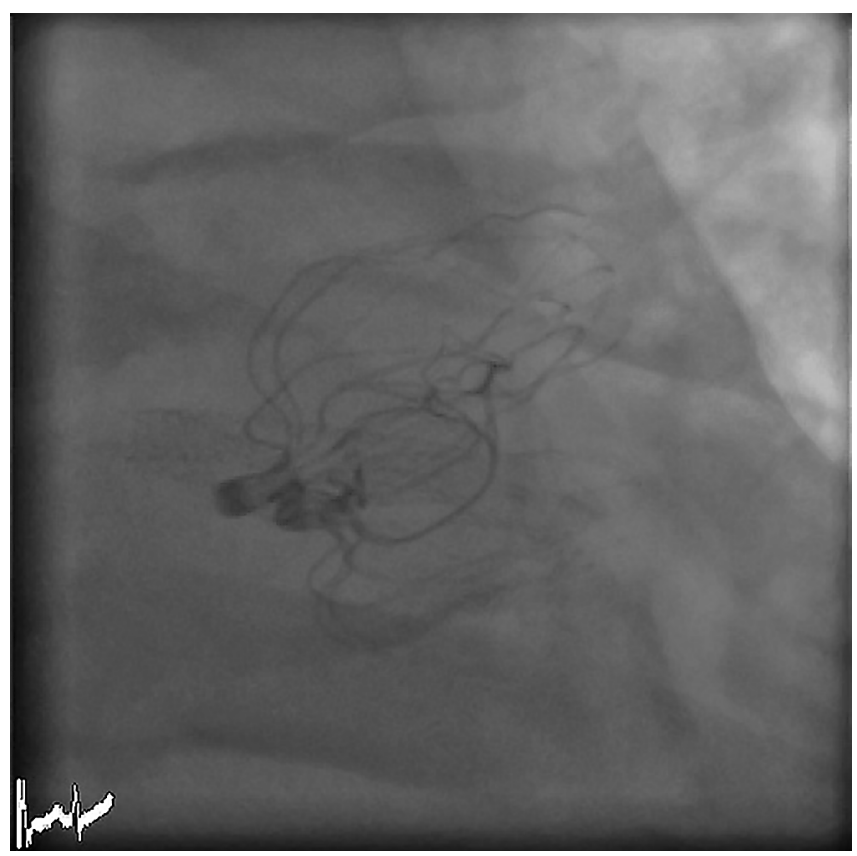

Video 5. Cine clip showing two WATCHMAN devices (Kissing WATCHMAN). View supplemental video at https://doi. org/10.12945/j.jshd.2019.010.18.sup.05.

studies, including PROTECT-AF [8], CAP Registry [9], PREVAIL [10], and ASAP studies [11], have proved the efficacy and safety of the Watchman device in different sets of patients. 


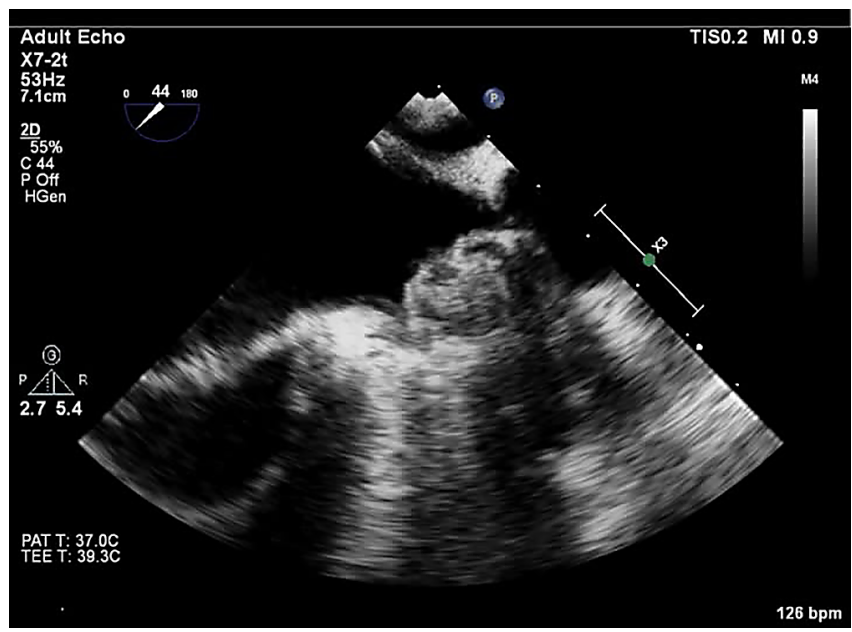

Video 6. TEE image showing well seated two WATCHMAN devices at 44 degrees. View supplemental video at https://doi. org/10.12945/j.jshd.2019.010.18.sup.06.

An autopsy study of the normal heart documented that $80 \%$ of the LAA have more than one lobe, with slightly more than half having 2 lobes [12]. Considering the great variability of the LAA anatomy in relation to size, shape, volume, number of lobes, and shape of the orifice, no single device is ideal to fit all [13]. The shape of the LAA ostium is elliptical in approximately $69 \%$ of cases, with a maximum depth ranging up to $51 \mathrm{~mm}$, while the rounded shape is present in only $5-6 \%$ of cases. The diameters of the ostium show minimal changes during the cardiac cycle (1-2 mm) and no change during AF [14]. Consequently, implanting a round device into an oval-shaped ostium may lead to incomplete occlusion and peri-device leakage. This problem is reported in $32 \%$ of the cases after Watchman implantation [15]. Incomplete occlusion of the LAA is thought to result in a higher event rate, but 2 analyses that used the PLAATO and Watchman systems, respectively, showed no increased event rate of thromboembolism [16]. Occasionally, if gaps are significant, then it is possible to occlude them by using different devices fully [17]. This eccentricity in the shape of the orifice also poses hurdles in estimating the exact size of the ostium by using two-dimensional (2-D) TEE and frequently results in an underestimation of the exact diameter, which leads to implantation of an undersized device.

In addition to the eccentric shape of the ostium, another problem related to the single-device clo- sure technique is the maximum body size of the LAA. Even the new-generation ACP and Watchman devices can fit into a maximum body diameter of $30 \mathrm{~mm}$ [18]. Exclusion of the LAA might require 2 devices in such cases. Enio et al. reported a case series where 5 of their patients underwent double-device implantation using devices other than Watchman, with good anatomical results at follow-up [3]. Implanting 2 Watchman devices in a single patient to close bilobulated LAA was previously reported once. The report concluded that occlusion of 2 separate lobes with a common ostium is practically possible, as the main bodies of each lobe are separated by a thick ridge of pectinate muscle [19].

Although it technically sounds feasible, few challenges are associated with double-device implantation. First, the polyethylene terephthalate membranes and nitinol cage covering the Watchman device can be damaged while releasing the second device, which itself can serve as a nidus for thrombus formation due to residual leakage and difficult endothelialization. Second, putting 2 round-shaped devices over an elliptical ostium can lead to multiple residual flows between the LA and LAA. Third, no long-term data are available to support double-device implantation in the LAA; therefore, delayed mechanical complications are unknown.

This case shows that sealing of a bilobed LAA is technically feasible especially with a favorable anatomy, which includes totally separated bodies of both lobes with adequate body sizes. Although this procedure can potentially result in damaging the delicate membranous part of the Watchman device, for the time being, no data are available to evaluate the long-term effects of this interaction. We also suggest that 2-D TEE alone can underestimate the size and anatomy of the LAA. Preprocedural assessment using three-dimensional TEE and intraprocedural angiography is crucial for better occlusion of the LAA.

\section{Conflict of Interest}

The authors have no conflict of interest relevant to this publication.

Comment on this Article or Ask a Question 


\section{References}

1. Wolf PA, Dawber TR, Thomas HE Jr, Kannel WB. Epidemiologic assessment of chronic atrial fibrillation and risk of stroke: the Framingham study. Neurology. 1978;28:973-977. DOI: 10.1212/ WNL.28.10.973

2. Hart RG, Pearce LA, Aguilar MI. Meta-analysis: antithrombotic therapy to prevent stroke in patients who have nonvalvular atrial fibrillation. Ann Intern Med. 2007;146:857-867. DOI: 10.7326/00034819-146-12-200706190-00007

3. Guerios EE, Gloekler S, Schmid M, Khattab A, Nietlispach F, Meier B. Double device left atrial appendage closure. Eurolntervention. 2015;11:470-476. DOI: 10.4244/ EIJY14M07_03

4. Camm AJ, Kirchhof P, Lip GY, Schotten U, Savelieva I, Ernst S, et al. Guidelines for the management of atrial fibrillation: the Task Force for the Management of Atrial Fibrillation of the European Society of Cardiology (ESC). Eur Heart J. 2010;31:2369-2429. DOI: 10.1093/eurheartj/ehq278

5. Sandercock P, Bamford J, Dennis J, Burn J, Slattery J, Jones L, et al. Atrial fibrillation and stroke: prevalence in different types of stroke and influence on early and long term prognosis (Oxfordshire Community Stroke Project). BMJ. 1992;305:1460-1465. DOI: $10.1136 / \mathrm{bmj} .305 .6867 .1460$

6. Blackshear JL, Odell JA. Appendage obliteration to reduce stroke in cardiac surgical patients with atrial fibrillation. Ann Thorac Surg. 1996;61:755-759. DOI: 10.1016/00034975(95)00887-X

7. Brass LM, Krumholz HM, Scinto JM, Radford M. Warfarin use among patients with atrial fibrillation. Stroke. 1997;28:2382-2389. DOI: 10.1161/01.STR.28.12.2382

8. Reddy VY, Doshi SK, Sievert H, Buchbinder $M$, Neuzil P, Huber $K$, et al. PROTECT AF Investigators. Percutaneous left atrial appendage closure for stroke prophylaxis in patients with atrial fibrillation: 2.3-year follow-up of the PROTECT AF (Watchman Left Atrial Appendage System for Embolic Protection in Patients with Atrial Fibrillation) Trial. Circulation. 2013;127:720-729. DOI: 10.1161/CIRCULATIONAHA.112.114389

9. Reddy VY, Holmes D, Doshi SK, et al. Safety of percutaneous left atrial appendage closure: results from the Watchman Left Atrial Appendage System for Embolic Protection in Patients with AF (PROTECT AF) clinical trial and the Continued Access Registry. Circulation. 2011;123:417-424. DOI: 10.1161/CIRCULATIONAHA.110.976449

10. Holmes DR Jr, Kar S, Price MJ, Whisenant B, Sievert H, Doshi SK, et al. Prospective randomized evaluation of the Watchman Left Atrial Appendage Closure device in patients with atrial fibrillation versus long-term warfarin therapy: the PREVAIL trial. J Am Coll Cardiol. 2014;8;64:1-12. DOI: 10.1016/j.jacc.2014.04.029

11. Reddy VY, Mobius-Winkler S, Miller MA, et al. Left Atrial Appendage Closure With the Watchman Device in Patients With a Contraindication for Oral Anticoagulation: The ASAP Study (ASA Plavix Feasibility Study With Watchman Left Atrial Appendage Closure Technology). J Am Coll Cardiol. 2013;61:2551-2556. DOI: 10.1016/j. jacc.2013.03.035

12. Veinot JP, Harrity PJ, Gentile F, Khandheria $\mathrm{BK}$, Bailey KR, Eickholt JT, et al. Anatomy of the normal left atrial appendage: $A$ quantitative study of age-related changes in 500 autopsy hearts: Implications for echocardiographic examination. Circulation 1997;96:3112-3115. DOI: 10.1161/01. CIR.96.9.3112

13. Hara H, Virmani R, Holmes DR, Buchbinder M, Lesser JR, Van Tassel RA, et al. Is left atrial appendage more than a simple appendage? Catheter Cardiovasc Interv. 2009;74:234-242. DOI: 10.1002/ccd.21983
14. Su P, McCarthy KP, Ho SY. Occluding the left atrial appendage: anatomical considerations, Heart. Vol. 94, no. 9, pp. 1166-1170, 2008. DOI: $10.1136 /$ hrt.2006.111989

15. Viles-Gonzalez JF, Kar S, Douglas P, Dukkipati S, Feldman T, Horton R, et al. The clinical impact of incomplete left atrial appendage closure with the Watchman device in patients with atrial fibrillation. J Am Coll Cardiol. 2012;59:923-929. DOI: 10.1016/j.jacc.2011.11.028

16. Viles-Gonzalez JF, Reddy VY, Petru J, Mraz T, Grossova Z, Kralovec S, Neuzil P. Incomplete occlusion of the left atrial appendage with the percutaneous left atrial appendage transcatheter occlusion device is not associated with increased risk of stroke. Journal of interventional cardiac electrophysiology : an international journal of arrhythmias and pacing. 2012;33:69-75. DOI: 10.1007/s10840-011-9613-x

17. Wunderlich N, Wilson N, Sievert, H. A novel approach to treat residual peridevice leakage after left atrial appendage closure. Cathet Cardiovasc Intervent. 2013;82:313319. DOI: $10.1002 / \mathrm{ccd} .23014$

18. Cruz-Gonzalez I, Yan BP, Lam YY. Left atrial appendage exclusion: state-of-the-art. Catheter Cardiovasc Interv. 2010;75:806813. DOI: $10.1002 / \mathrm{ccd} .22344$

19. Liu Z, Yu J, Fang PH, He J, Wang JD, Wang $\mathrm{H}$, et al. Double device left atrial appendage closure with the WATCHMAN ${ }^{\text {TM }}$. Int J Cardiol. 2015;187:281-282. DOI: 10.1016/j. ijcard.2015.03.289

Cite this article as: Alsmadi $F$, Shah MA, Qattea MB. Left Atrial Appendage Closure with Double Watchman Devices: A Case Report. Structural Heart Disease. 2019;5(1):1-6. DOI: https://doi. org/10.12945/j.jshd.2019.010.18 